\title{
Conceptual design of the ALADDIN Antarctic nulling interferometer
}

\author{
Marc Barillot $^{a}$, Pascal Courteau ${ }^{a}$, Olivier Absil ${ }^{b}$, Vincent Coudé du Foresto ${ }^{c}$, and Mark Swain ${ }^{d}$ \\ ${ }^{a}$ Alcatel Alenia Space ; 100, Bd. Du Midi, F-06156 Cannes La Bocca Cedex, France ${ }^{1}$ \\ ${ }^{b}$ Université de Liège ; Allée du 6 Aout, 17, B5C, B-4000 Sart Tilman, Belgium \\ ${ }^{c}$ Observatoire de Paris-LESIA ; 5, place Jules Janssen, 92195 Meudon Cedex, France \\ ${ }^{d}$ Max-Planck Institüt für Astronomie ; Königstuhl 17, D-69117 Heidelberg, Germany
}

\begin{abstract}
It is commonly accepted that highly challenging planet finding missions such as Darwin and TPF need precursors on the ground, for both technological demonstration and study of the exozodiacal clouds around potential targets. A first instrument, GENIE, designed to be implemented in the interferometric laboratory of the VLTI, was studied by ESA and scientific/industrial teams. In this paper we present a concept for ALADDIN, an operational nulling instrument to be implemented at Dome C in Antarctica, and discuss the comparison with GENIE from the instrumental point of view. Our preliminary design involves moderate $\sim 1 \mathrm{~m}$ size telescopes mounted on a $40 \mathrm{~m}$ long rotating beam allowing baselines up to $30 \mathrm{~m}$ and feeding a 2 -arm nulling beam combiner. When compared to GENIE, the rotating beam design has the advantage of removing the need for both long-stroke delay line and dispersion control equipments. As a side effect, the instrumental arrangement of ALADDIN finds itself more representative of what Darwin will be. Furthermore, critical issues like phase control, photometric balance and instrumental background suppression are expected to be relaxed by the improved atmospheric conditions, lower temperature, and simpler optical trains. Calibration of geometrical stellar leakage will make advantage of the continuously adjustable baseline. As results, a simpler instrument showing improved performance is expected. In conclusion, we see our ALADDIN concept as a valuable alternative to GENIE, with a quite stronger scientific potential and a considerably simplified instrumental design.
\end{abstract}

Keywords: Darwin, TPF, GENIE, interferometry, nulling, Antarctica, optics, instrumentation, astronomy

\section{INTRODUCTION}

The search for Earth-like exoplanets, which orbit in the habitable zone of stars other than our Sun and show biological activity, is one of the most exciting and challenging quests of the present time. Nulling interferometry from space, in the thermal infrared, appears as a promising candidate technique for the task. It has been studied for about 10 years by ESA and NASA in the framework of the Darwin and TPF-I missions respectively ${ }^{1}$.

Nevertheless, the challenge is such that the research and development effort is expected to require several more years of investigations at all levels, system analysis, technology development, and experimentation. Precursor missions are expected to help refining the science mission, through in particular the direct observation of exozodiacal light and giant planets, and demonstrate the technological progress on an operational basis. Several precursor missions have been introduced, either in space; such as the Fourier-Kelvin Stellar Interferometer ${ }^{2}$ and PEGASE ${ }^{3}$, or on the ground, such as the Keck Interferometer Nuller ${ }^{4}$ and the Darwin-GENIE experiment ${ }^{5}$.

A cooperation between ESA and ESO, the Darwin-GENIE mission aims at taking advantage of ESO's Very Large Telescope Interferometer (VLTI) infrastructure, which offers a huge collecting area, cutting-edge functionality for interferometry, and an operational environment.

1 Marc Barillot, telephone: 33 (0)4 92927963 ; fax: 33 (0)4 92927160 ; email: marc.barillot@alcatelaleniaspace.com Pascal Courteau, telephone: 33 (0)4 92926625 ; fax: 33 (0)4 92927160 ; email: pascal.courteau@alcatelaleniaspace.com Olivier Absil, telephone: 32 (0)4 3669766 ; fax: 32 (0)4 3669746 ; email: olivier.absil@ulg.ac.be

Vincent Coudé du Foresto, telephone: 33 (0)1 45077961 ; fax: 33 (0)1 45077102 ; email: vincent.foresto@obspm.fr Mark Swain, telephone: (+49 6221) 528 - 0 ; fax: (+49 6221) 528 - 246 ; email: swain@ mpia.de 
An Alcatel Alenia Space-led team involving several of the authors of the present paper (Barillot, Courteau, Absil and Coudé du Foresto) was involved in a Definition Phase study of GENIE for ESA, which contributed to the demonstration of the potential of the experiment to detect exozodiacal dust clouds down to 30-60 times the optical thickness of the Solar system's ${ }^{6}$. However, to achieve that performance requires a relatively complex optical layout (see Figure 1) as well as sophisticated and accurate real-time controls and calibration procedures, in order to compensate for the atmosphereand VLTI-induced disturbances (turbulence, background, etc...) and to remove the geometrical stellar leaks. As results, the achievement of the ultimate performance potential of GENIE would generate substantial progress in the nulling technology, at the cost of a quite significant development effort.

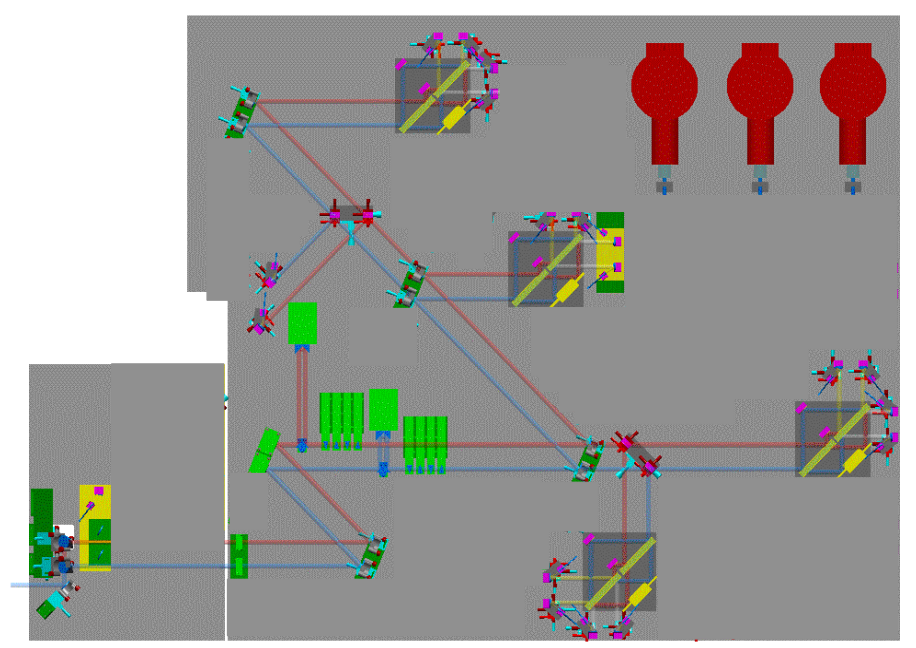

Figure 1: Preliminary optical layout of GENIE, in its simplest "single-Bracewell" variant.

Based on the statement that several of the most stringent instrumental requirements of GENIE would be significantly relaxed in quieter atmospheric conditions, and on the emergence of the Dome $\mathrm{C}$ site in Antarctica as possibly the best seeing on Earth ${ }^{7,8,9}$, the idea of building a nulling interferometry experiment at Dome $\mathrm{C}$ was proposed recently ${ }^{10}$ under the acronym ALADDIN.

Guidelines specific to the ALADDIN mission are introduced in Section 2. Then, our preliminary approach on the ALADDIN conceptual design follows in Section 3.

\section{SPECIFIC ALADDIN GUIDELINES}

\subsection{Specificity of the Antarctic location}

As far as temperature and humidity are concerned, the Antarctic environment is expected to be very well compatible with the development of high-performance optical instrumentation, through the respect of rules inspired from the development of cryogenic optics, and with the help of protective solutions such as those used in space for components sensitive to the condensation of ice and contaminants. By some respects, the low and stable temperature and the dryness of the air is considered as favorable conditions for infra-red interferometry.

According to recent characterizations of the dome $\mathrm{C}$ site ${ }^{11}$, the Antarctic seeing is excellent above a $36 \mathrm{~m}$ thick ground layer. As results, the collecting optics should be accommodated 36m above the ground surface.

A third feature of the Antarctic environment is human intervention is far more difficult than in temperate sites. Therefore ALADDIN should be able of nearly automatic operation. Manual adjustments and calibrations should be minimized. In order to do so, the experiment design should be simple, i.e. based on moderate diameter telescopes, and involve as few components and as little active functions and mechanical actuators as possible. In particular, ALADDIN will include not more than 2 interferometric arms. In the same spirit, emphasis should be put on the reliability of the equipment, which also benefits from a reduced number of active functions. 
As an important consequence, thorough, end-to end functional validations of the equipment and of all the assembly, adjustment, calibration and operation procedures should be achieved in a temperate location, before the whole apparatus is sent to Dome C. This strongly argues in favor of an autonomous and dedicated mission, which will be free from the inevitable complexity of design and operation of a multi-purpose instrument, and of the validation of the interfaces with a multi-purpose infrastructure.

Finally, the fact that there is currently no or almost no available infrastructure for astrophysics at Dome $\mathrm{C}$ also plays in favor of an autonomous concept and completion of end to end tests before transfer to the Antarctic site.

\subsection{Performance issues}

As already mentioned, the performance goal of ALADDIN is quite challenging. From the GENIE experience, and making use of the same GENIEsim ${ }^{6}$ simulator software as used for GENIE, we may list the following critical performance aspects.

As for GENIE, the selected operating wavelength is the L, or L' band, which represents a good compromise, in our opinion, between the thermal background, turbulence effects versus wavelength, and contrast between the star and the surrounding disk or companion(s).

The instrument must be able of deep nulling (rejection ratio above 10000) in a stable way. However, thanks to the low atmospheric turbulence, real-time control loops are limited to tip-tilt control and phase stabilization. Due to longer coherence time when compared to a temperate site, they can be run at lower closed loop frequencies. Besides, real-time control loop of dispersion compensation, which requires critical hardware development while being not relevant for a DARWIN/TPF demonstration, is avoided by the selection of a rotating beam architecture (detailed in section 3). Deep nulling also requires full symmetry of the optical design, for polarization matching reasons.

The instrument must provide very efficient background calibration, given the very weak signal level of the science targets. We found transmission efficiency as high as typically $8 \%$, including a $65 \%$ quantum efficiency detector, can be achieved by means of the reduction of the number of optical surfaces with respect to a conventional design. This, together with an operation at the ambient temperature of $210 \mathrm{~K}$, allows the reduction of the background to a level compatible with the mission. Indeed, ultimate reduction of the number of surfaces to 9 can be achieved, again by means of the rotating beam architecture: coudé trains, as well as long range delay lines, can be removed from the optical paths.

As results, thanks to the optimization of the transmission efficiency and the relatively moderate loop frequencies, collecting telescope diameters of $1 \mathrm{~m}$ provide enough photons to feed the active controls, as well as detect science signal with enough SNR.

The instrument must provide very efficient geometrical leakage calibration. Adjustable baselines in the range 4-30m provide an additional means of calibrating the geometrical leakage, by comparing the leaks for various baselines, and allow the selection of the most efficient resolution power for the long duration observations.

\subsection{Development issues}

As a pathfinder for Darwin/TPF, ALADDIN must be able of a fast and autonomous development, in order to be independent from the existence or not of an infrastructure at Dome C. In the same spirit, it can be dismantled immediately after having completed its main mission, thus leaving the space, and possibly pieces of infrastructure, available for other instruments. It must also rely on available technology, which is again in favor of L band operation. The required simplicity of the design, limited number of equipment and functions, and relaxation of real-time control requirements, also consolidate the development schedule.

Furthermore, the development of the supporting structure, optical trains, and nulling instrument can be parallelized. This, combined with the possibility to validate the whole system in a temperate location, provides additional mitigation of the risks. 


\section{DESCRIPTION}

Based on the guidelines above, the overview of our conceptual design for ALADDIN is illustrated in Figure 2.

\section{Telescopes assemblies}

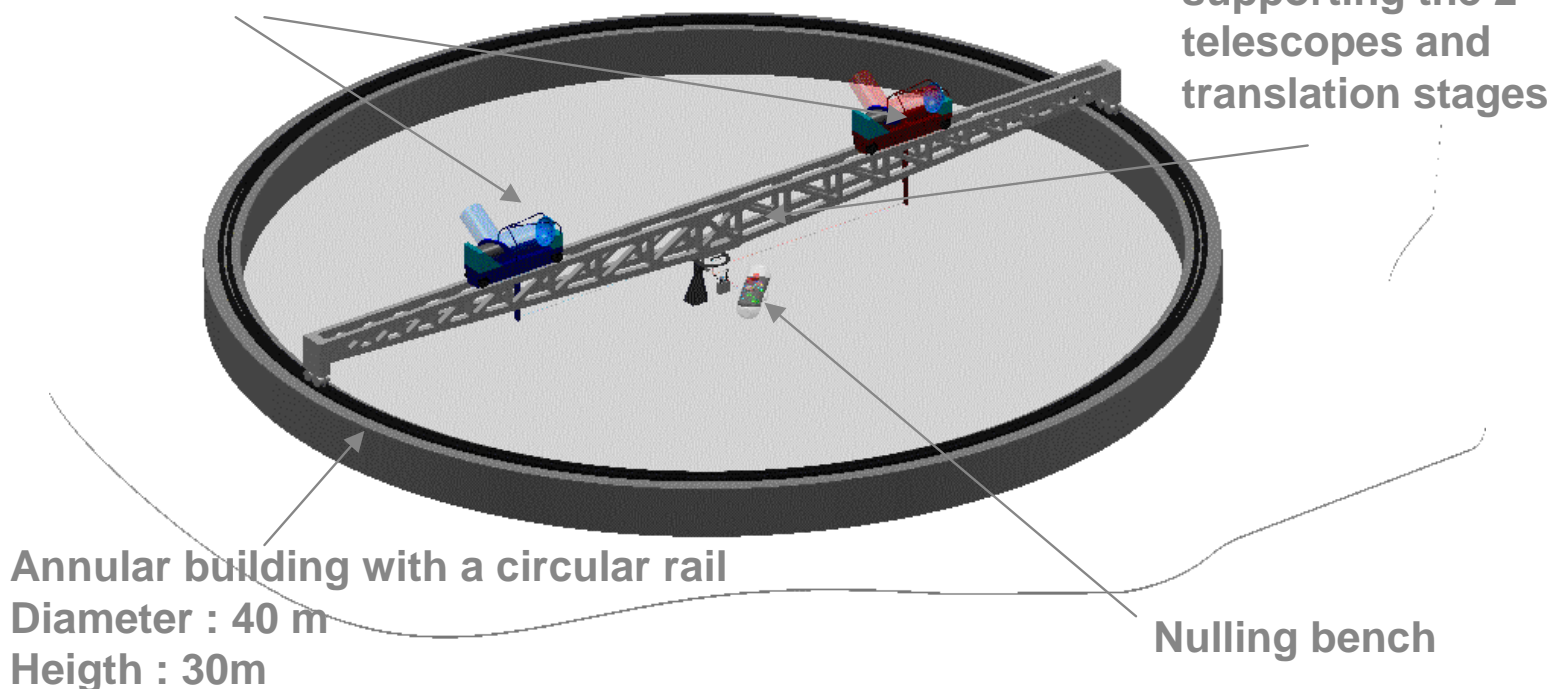

Figure 2: Overview of the ALADDIN experiment

ALADDIN is a fully autonomous observation system. It features two $1 \mathrm{~m}$ diameter collectors accommodated on a straight $40 \mathrm{~m}$ long structural beam. The latter can be oriented in azimuth so that the source is maintained at the meridian of the baseline. Collectors are plane-mirror siderostats, and can be adjusted to the elevation of the target. Off-axis telescopes provide compression of the light beams down to typically $40 \mathrm{~mm}$. The collectors can slide along the structural beam (but remain fixed during observations) so that the $4-30 \mathrm{~m}$ baseline (and stellar leaks) can be adjusted according to the observed target.

The siderostats, telescopes and translation stages are shown in Figure 3.

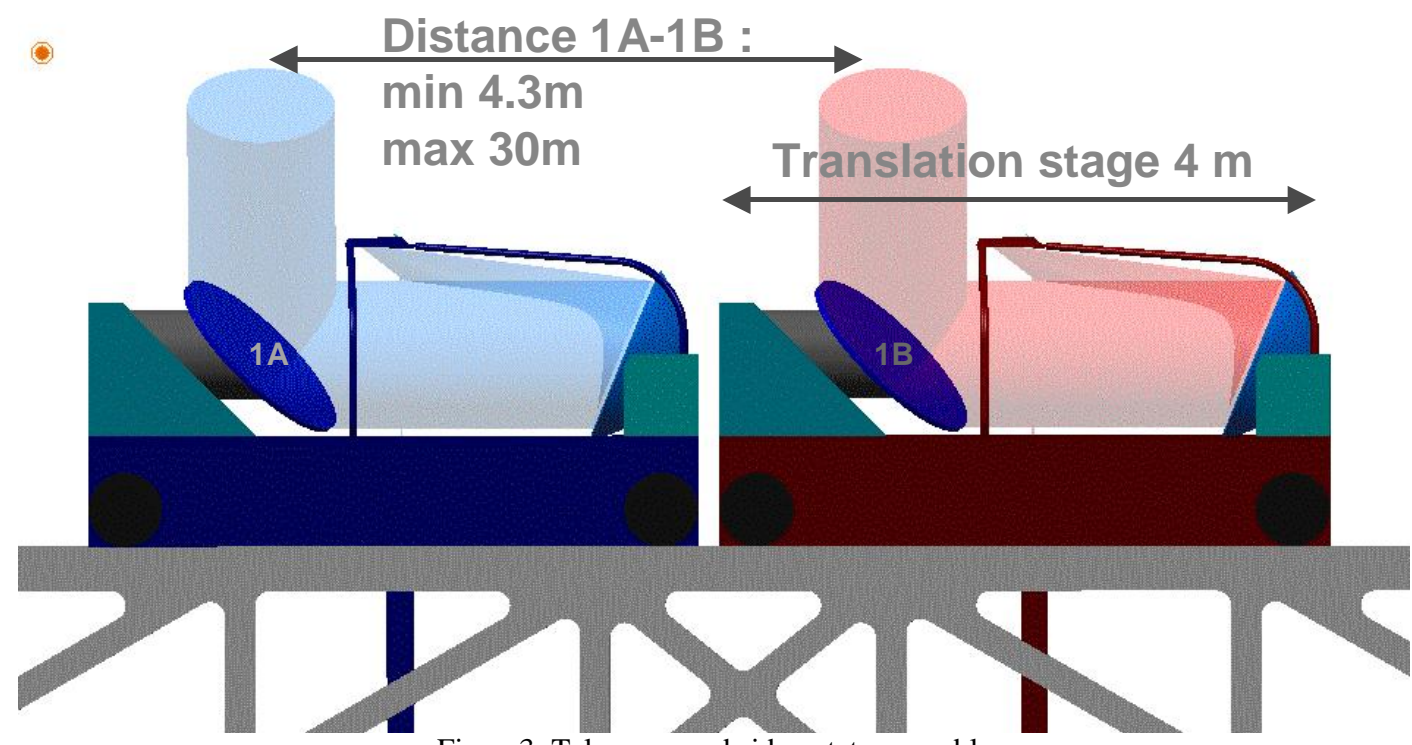

Figure3: Telescope and siderostats assembly 
The compressed light beams are then tip-tilt corrected and de-rotated by means of a simple sequence of plane mirrors, so that the rest of the optical paths may remain immobile with respect to the ground. In particular, the nulling bench, shown in Figure 4, is mechanically de-coupled to the rotating beam so that the stability of the instrument components, which lie downstream the Optical Path Difference (OPD) and tip-tilt sensors, is preserved. Thanks to the fully symmetrical arrangement of the telescopes, light beam combination remains perfectly symmetric whatever the rotation angle of the structural beam with respect to the nulling instrument.

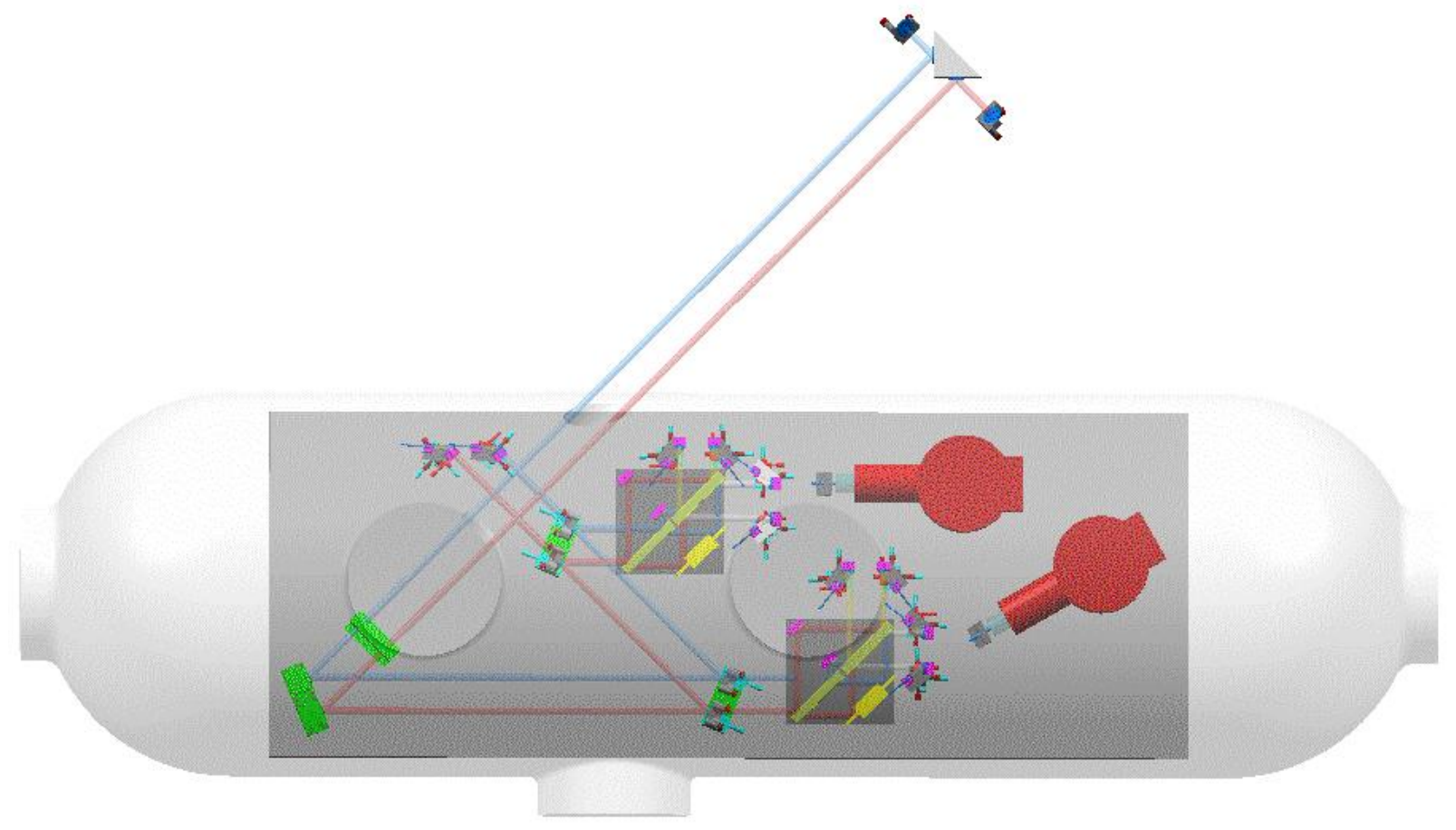

Figure 4: Nulling instrument, shown here enclosed in a cryostat.

There is no long stroke delay line thanks to the continuous or step-by-step angular adjustment of the structural beam, so that the source is always maintained at the meridian of the baseline. Only a short-stroke delay line is required to compensate for OPD fluctuations generated by differential turbulence and mechanical vibrations. Assuming the latter can be kept small enough, a simple reflection on a plane mirror, close to normal incidence, is enough for this task, with the important advantage of being able to meet the actuation frequency (on a G0V star at 20pc GENIEsim calculations show that a $4 \mathrm{kHz}$ loop rate is sufficient to maintain the piston residuals below $13 \mathrm{~nm}$ rms, using photons from the $\mathrm{K}$ band only).

Achromatic Pi-phase shifting is geometrically introduced by means of a pair of mirror-symmetric right angle periscopes $^{12}$. At the same time, the periscopes allow the adjustment of the spacing between the light beams at the input of the nulling bench, and bring a degree of freedom for coarse adjustment of the OPD if necessary, e.g. for occasional de-saturation of the short-stroke delay line, since the telescope translating stages are likely to remain locked during observation for stability reasons.

The nulling bench $(2.4 \times 0.9 \mathrm{~m})$ is located in a cryostat for lower thermal emission and better stability. In a first approach, it consists of a simplified single-Bracewell version of GENIE (Figure 1). In particular, the dispersion compensation and intensity control loops have been removed, thanks to the very stable atmosphere at Dome C. As a side effect, the possible coupling between the intensity and OPD controls disappears. 
Thanks to the superior transparency of the atmosphere, the wavelength coverage of the science channel can be as large as 3.1-4.1 $\mu \mathrm{m}$, whereas the $\mathrm{K}$ band throughput is sufficient for fringe tracking. In Figure 3, two Modified Mach-Zender ${ }^{13}$ (MMZ) beam combiners are present, one for the science channel in the L band, the other one for OPD tracking in the $\mathrm{K}$ band, with the appropriate adjustment to enable the implementation of an accurate phase sensing algorithm. An alternative arrangement where the two functions share one single beam combiner can also be envisaged for better stability, as discussed in subsection 4.2.

\section{DISCUSSION}

\subsection{Rotating beam or conventional design ?}

The present design is strongly driven by the selection of the rotating beam design rather than the more conventional architecture, which involves independent telescopes connected by optical tunnels. Such a choice was primarily justified by performance considerations.

Performance arguments in favor of the rotating beam include:

- the fully symmetrical optical paths, and in particular the identical atmospheric thickness in the two arms whatever the location of the target in the sky, avoids longitudinal dispersion and the need for a dedicated realtime control, long-stroke delay lines, and plays in favor of best polarization matching,

- the reduction of the number of optical surfaces by avoiding the use of Coudé trains and long-stroke delay lines improves the transmission of the instrument and reduces the level of background

- the calibration capabilities of ALADDIN are improved by the possibility to invert the collectors for a given baseline length: rotating the truss by $180^{\circ}$ provides additional information on the symmetry of the instrument.

Furthermore, the rotating beam design needs less actuators and controls than the conventional design, and as such is expected to be operationally more practical and reliable, either in tests or during science observations.

A question lies in the practical assembly and integration of a 40m long structural beam at a $36 \mathrm{~m}$ altitude at Dome C. Actually, the size of the structure is very comparable to the dimensions of the structure of a UT telescope of the VLTI. The fabrication of such a structure can therefore be considered feasible in a first approach. Besides, it seems a priori more practical than building two conventional telescopes on top of $36 \mathrm{~m}$ high structures and connecting them with optical tunnels, particularly if the towers are to be moveable so that the baseline is adjustable. A single, static circular structure also seems to have more potential for geometrical stability than the sum of two separate moveable structures.

Integrating such a structure at Dome $\mathrm{C}$ will be an innovative situation. Nevertheless, one can arguably defend an optimistic position, and state that according to Storey ${ }^{13}$ "while the idea of constructing a large astronomical facility in Antarctica may sound challenging, over a decade of experience at South Pole Station suggests that it is no more difficult than - just different from - constructing a telescope to operate at a temperate location". That said, we do not pretend the development of ALADDIN will be straightforward. In particular, the limited experience of the community in terms of integrating complex optical systems in Antarctica has undoubtedly to be taken into account. The possible impact of the structure on the turbulence should also be kept in mind. On both respects, appropriate learning activities ought to be performed before ALADDIN is transferred on the site.

\subsection{Open optical issues}

The present paper describes the broad outline of the optical architecture of ALADDIN. Several topics remain to be studied more in details. Among others, the afocal telescope magnification, the question of pupil location and possible imaging, the location of the tip-tilt sensor, the need to enclose the instrument in a cryostat and the impact of the cryostat windows on the nulling, the need to implement a static dispersion corrector to cope with possible dispersion at modal filtering level, etc..., need some revisiting.

Our current design of the nulling bench includes the delay line for simplicity and commonality with GENIE. However an alternative implementation at the level of the tip-tilt mirror is worth envisaging, since it would remove the last mechanically active equipment from the nulling bench. Moreover, it might allow further reduction of the number of 
actuators by one, as well as suppress one reflection, if it is confirmed the tip-tilt and delay line actuators could be provided by the same component.

At the nulling level, an attractive alternative would be to use the same components for both MMZ, e.g. with a vertical separation of the two channels and a L-K spectral separation in the vertical rather than the horizontal plane. The resulting layout would reduce non-common paths, be extremely compact, hence stable, and would require a smaller cryostat.

\section{CONCLUSION}

In this paper, we have presented an overview of our conceptual design of the ALADDIN nulling instrument for Antarctica. ALADDIN features two $1 \mathrm{~m}$ telescopes mounted on a rotating beam, which feed a quite simple nulling interferometer where only the short-stroke delay line and tip-tilt need to be controlled in real-time.

ALADDIN is a stand-alone experiment; independent from any existing observational infrastructure, and dedicated to one mission, precursor science and technology for Darwin and TPF, with a limited operational duration. As such, and thanks to the specific sensitivity of nulling interferometry, ALADDIN is quite well suited for an early operational characterization of the Dome C observing site.

As far as the preparation of Darwin is concerned, ALADDIN appears as a complementary approach with respect to Darwin-GENIE, for two reasons. First, while the technological challenge of the latter lies in achieving accurate stabilization and calibration of a very complex nulling instrument, the former focuses on integrating an autonomous nulling experiment in an unusual environment, with an interesting commonality with space in the sense that operation with minimum human intervention is required. Second, despite the similarity of the missions, the architecture of both experiments are radically different, and can be expected to provide complementary technological outcomes.

As results we consider ALADDIN to be a potentially rich source of not only scientific but also instrumental progress on the way to Darwin and TPF. Either from its innovative design, or from its unusual location, on the overall the ALADDIN challenge shows many attractive aspects, and in our opinion deserves to be studied further.

\section{REFERENCES}

1. Fridlund, Malcolm C., "Darwin and TPF: technology and prospects", Proceedings of the SPIE, Volume 5491, pp. 227-235, 2004.

2. Richard Barry, William Danchi, Jayadev Rajagopal, Victor John Chambers, Lee Jeremy Richardson, Anthony Martino, Ronald Allen, Marc Kuchner, Sara Seager, Wesley Traub, Debra Wallace, "The Fourier-Kelvin Stellar Interferometer: an achievable, space-borne interferometer for the direct detection and study of extrasolar giant planets" , Proceedings of the IAU, Coll. N²00, pp. 221-226, 2005

3. Marc Ollivier, Jean-Michel Le Duigou, Denis Mourard, Olivier Absil, Frédéric Cassaing, Emilie Herwats, Laurent Escarrat, Bruno Chazelas, France Allard, Rodolphe Clédassou, Vincent Coudé du Foresto, Michel Delpech, Paul Duchon, Pierre-Yves. Guidotti, Alain Léger, Xavier Leyre, Fabien Malbet, Daniel Rouan, Stéphane Udry, "PEGASE : a TPF/DARWIN pathfinder", Proceedings of the IAU, Coll. N²00, pp. 241-246, 2005

4. Bertrand Mennesson, R. Akeson, E. Appleby, J. Bell, A. Booth, M.M. Colavita, S. Crawford, M.J. CreechEakman, W. Dahl, J. Fanson, C. Felizardo, J. Garcia, J. Gathright, J. Herstein, E. Hovland, M. Hrynevych, E. Johansson, C. Koresko, D. Le Mignant, R. Ligon, R. Millan-Gabet, J. Moore, C. Neyman, D. Palmer, T. Panteleeva, C. Paine, S. Ragland, L. Reder, A. Rudeen, T. Saloga, E. Serabyn, M. Shao, R. Smythe, K. Summers, M. Swain, K. Tsubota, C. Tyau, G. Vasisht, P. Wizinowich, J. Woillez, "Long Baseline Nulling Interferometry with the Keck Telescopes: a Progress Report", Proceedings of the IAU, Coll. N²00, pp. 227-232, 2005

5. Gondoin, Philippe A.; Absil, Olivier; den Hartog, Roland H.; Wilhelm, Rainer C.; Gitton, Philippe B.; d'Arcio, Luigi L.; Fabry, Pierre; Puech, Florence; Fridlund, Malcolm C.; Schoeller, Markus; Glindemann, Andreas; Bakker, Eric J.; Karlsson, Anders L.; Peacock, Anthony J.; Volonte, S.; Paresce, Francesco; Richichi, Andrea, " DarwinGENIE: a nulling instrument at the VLTI", Proceedings of the SPIE, Volume 5491, pp. 775-786, 2004.

6. O. Absil, R. den Hartog, P. Gondoin, P. Fabry, R. Wilhelm, P. Gitton and F. Puech, "Performance study of groundbased infrared Bracewell interferometers", $A \& A$ 448, pp; 787-800, 2006. 
7. LLoyd, J., Lane, B., Swain, M., Storey, J., et al., "Extrasolar Planet Science with the Antarctic Planet Interferometer", Proceedings of the SPIE, Volume 5170, pp. 193-199, 2003.

8. Swain, M., Coudé du Foresto, V., Fossat, E. and Vakili, F, "The Antarctic Planet Interferometer and the Potential for Interferometric Observations of Extrasolar Planets from Dome C Antarctica", Mem. S.A.It. Suppl. Vol. 2, 207, 2003.

9. M. R. Swain et al., " The Antarctic Plateau Interferometer Executive White Paper", EAS Publications Series, Vol. 14, M. Giard, F. Casoli and F. Paletou (eds), pp. 147-156, 2005

10. Coude du Foresto, Vincent; Absil, Olivier; Vakili, Farrokh, and Swain, Mark R., "The Antarctic plateau as a site for a Darwin ground-based precursor", Proceedings of Visions for Infrared Astronomy (VIRA), to be published, 2006

11. Agabi A., Aristidi E., Azouit M., Fossat E., Martin F., Sadibekova T., Vernin J., Ziad A., "First Whole Atmosphere Nighttime Seeing Measurements at Dome C, Antarctica", Publ. Astron. Soc. Pacific 118, pp. 344-348, 2006

12. E. Serabyn, M. M. Colavita, "Fully Symmetric Nulling Beam Combiners", Applied Optics, Vol. 40, Issue 10, pp. $1668-1671,2001$

13. J.W.V. Storey, " Antarctica: the potential for interferometry", Proceedings of the SPIE, Volume 5491, pp. 169$175,2004$. 